\title{
Über die Beziehung der auf Lichtreize hin auftretenden bioelektrischen Erscheinungen der Grosshirnrinde des Menschen zur Reaktionszeit.
}

\author{
Von \\ Tosisada Mita. \\ (三田俊定) \\ (Aus dem II. Physiologischen Institut der Tohoku \\ Kaiserlichen Universität zu Sendai. \\ Vorstand: Prof. Dr. K. Motokawa.)
}

\section{Einleitung.}

Seit der Bergerschen Entdeckung (1929) der elektrenkephalographisch erkennbaren Grosshirnwellen ist von mehreren Autoren nachgewiesen worden, dass die bioelektrischen Erscheinungen der Grosshirnrinde bei der Belichtung des Auges eine Veränderung erfahren, insbesondere dass die $\alpha$-Wellen durch Lichtreize unterdrückt werden.

Wenn die auf Lichtreize möglichst rasch antwortende motorische Reaktion im zeitlich bestimmten Zusammenhang mit den auf dieselben Reize auftretenden bioelektrischen Veränderungen der Grosshirnrinde gebracht wird, so wird es nicht nur zur Deutung des Elektrenkephalogramms (E.E.G.) sondern auch zur vergleichenden Untersuchung des letzteren mit der subjektiven Empfindung wesentlich beitragen. Einige Forscher, Durup, ${ }^{3)}$ Loomis, ${ }^{\text {9) }}$ Jasper und Cruikshank ${ }^{6}$ ) u. a., haben die Latenzzeit der $\alpha$-Wellen-Unterdrückung und auch die Reaktionszeit gleichzeitig gemessen und mitgeteilt, dass die Reaktionszeit, wie erwartet, überhaupt länger als die Latenzzeit der $\alpha$ Wellen-Unterdrückung war.* Aber bei diesen Versuchen ist die Beziehung zwischen der Reaktionszeit und der Latenzzeit der $\alpha$ Wellen-Unterdrückung nur einer bestimmten Lichtstärke und zwar ohne Rücksicht auf den Adaptationszustand des Auges untersucht

* Aber das vollkommene Verschwinden der $\alpha$-Wellen war nicht immer kürzer als dis Reaktionszeit. 
worden. Also habe ich unternommen, die Beziehung der motorischen Reaktion auf Reizlicht zu den bioelektrischen Erscheinungen der Grosshirnrinde sowie deren Abhängigkeit von der Reizstärke bei dem bestimmten Hell- und Dunkeladaptationszustand noch systematischer zu untersuchen. Ich habe natürlich die Reaktionszeit und die bioelektrischen Veränderungen miteinander auf demselben Aufnahmepapier registriert, und aus dem so aufgenommenen E.E.G. verschiedenenartige Latenzzeiten an Hand der verschiedenen Merkmale des elek- . trischen Bildes gemessen, um sie mit der Reaktionszeit zu vergleichen.

Es ist selbstverständlich, dass sich der Zeitpunkt der $a$-WellenUnterdrückung um so sicherer und richtiger bestimmen lässt, je grösser die Verstärkung und die Stabilität des Verstärkers sind. Daher bemühte ich mich zuerst um die Herstellung eines zu diesem $Z$ wecke dienenden Verstärkers.

Ich danke Herrn Prof. M. Matsudaira vom elektrotechnischen Institut der hiesigen Universität herzlich für die freundliche Leitung und Unterstützung bei der Verfertigung des Verstärkers.

\section{Methodik.}

\section{A. Elektrode und Ableitungsweise.}

Zur Registrierung des E.E.G. wurde die bipolare Ableitung verwendet. Die Elektroden bestanden aus reinen Silberplättchen der Grösse von $1 \times 10 \times 20$ mm mit einem Utberzug aus Gase, die mit Kochsalzlösung getränkt war. Die eine der beiden wurde an dem Hinterhaupthöcker und die andere in der Mitte der Stirn mit einem Gummiband festangelegt. Die Hinterhaupt elektrode wurde zum Gitterpol der ersten Stufe des Verstärkers mit dem geschirmten Draht zugeleitet, die Stimelektrode zum Erdpol.

\section{B. Verstärker.}

Ich habe einen vierstufigen Verstärker verwendet, dessen elektrische Leitschaltung schematisch in Abb. 1 angezeigt ist. Dieser Verstärker hat, abgesehen von der ersten Stufe, die prinzipiell gleiche Verbindungsschaltung wie derjenige, mit welchem I to und $\mathrm{Ka} \mathrm{keta^{8 }}$ fruther in diesem Institut den Grosshirnaktionsstrom untersuchten. Ich habe diesen Verstärker noch verbessert, um eine höhere Verstärkung sowie eine bessere Stabilität zu erzielen.

Die verbesserten Punkte sind folgende.

1. Alle Stromquellen der ersten Röhre, d.h. Betriebs-, Heizfaden- und Anodenspannungsbatterie wurden von den der nächsten drei Röhren getreñnt geschaltet. 
2. Zur Vermeidung der eventuellen Rückkopplung wurde die vierte Röhre (UX-71 A) auch mit einer selbständigen Anodenbatterie versorgt.

3. Die geeignetesten Schirmgitterspannungen für die 3 Röhren (UZ-77) wurden bésonders sorgfältig gewählt, indem die Abhängigkeit der Verstärkung von der Schirmgitterspannung je an einzelnen Röhren durch die gewöhnliche Methode mit einem Schwebungssummer und einem Röhrengalvanometer geprüft wurde, weil die ge: eigneteste Schirmgitterspannung selbst bei Röhren von gleicher Art von Röhre zu Röhre verschieden sein konnte.

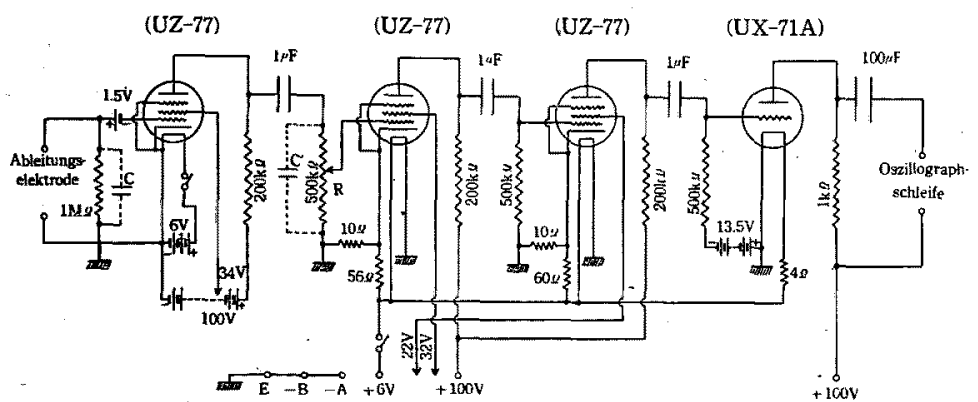

Abb. 1. Schaltungsschema des Verstärkers.

Die Verstärkung-Frequenz-Kennlinie dieses Verstärkers verläuft ungefähr gleichmässig von 10 bis $1000 \mathrm{~Hz}$. Nach der wirklichen Messung mit 50 hertzigem Wechselstrom betrug die maximale Spannungsverstärkung des Verstärkers $5 \times 10^{4}$ bei einem 100 ohmigen Belastungswiderstand. Bei dem inneren Widerstand der Oszillographschleife von $2 \mathrm{Ohm}$ reduzierte sich die Spannungsverstärkung zu ungefähr $10^{3}$. Diese Verstärkung war für die Aufnahme des E.E.G. sogar zu gross, weil die Empfindlichkeit der gebrauchten Oszillographschleife $10^{-4} \mathrm{~V} / \mathrm{mm}$ war. Also musste die Verstärkung durch Regulierwiderstand $(\mathrm{R})$ passend herabgesetzt werden. In der Praxis schaltete ich ferner im letzten Anodenkreis einen Widerstand von $1000 \mathrm{Ohm}$ parallel zum Oszillographschleifenkreis ein, in welchem andererseits ein Elektrolytenkondensator von $100 \mu \mathrm{F}$. eingeschaltet wurde, wie in Abb. 1 ersichtich ist, so dass die Gleichstromkomponente vom Oszillographschleifenkreis ausgeschaltet und die unangenehme zu starke Abweichung der Nullinie vermieden wurde.

Da es bei meiner Untersuchung nur darauf ankommt, die $\alpha$-Wellen sowie die relativ langsame Spannungsschwankungen darzustellen, habe ich versucht, die anderen hochfrequenten kleinen Wellen ab- 
zufiltrieren. $\mathrm{Zu}$ diesem $\mathrm{Zweck}$ wurden Kondensatoren von $1 \mu \mathrm{F}$ und $0,3 \mu \mathrm{F}\left(\mathrm{C}\right.$ und $\mathrm{C}^{\prime}$ in $\left.\mathrm{Abb} .1\right)$ im Gitterkreise der ersten und der zweiten Röhre resp. den Gitterwiderständen nebeneinander eingeschaltet. Durch diese Massnahme wurde die sonst gleichförmige Kennlinie derart umgestaltet, wie in Abb. 2 dargestellt ist. . Die durch diesen Verstärker aufgenommenen E.E.G. sind beispielsweise in Abb. 5 und 8 wiedergegeben.

Die geringe Verzerrung der Wellenform infolge der Kondensatoreneinschaltung ist bei meiner Untersuchung fast belanglos, da es hauptsächlich auf die Unterdrückung der $\alpha$-Wellen ankommt, vielmehr bringt es den Vorteil mit sich, dass die Veränderung dieser Wellen, ohne durch andere hochfrequente Wellen gestört zu werden, sehr leicht erkennbar ist.

Der andere Vorteil ist, dass durch diese Kondensatoreneinschaltung sowohl die hochfrequenten Fremdeinflüsse, wie z.B. Stadtstrom, als auch die Geräusche der Elektronenröhre des Verstärkers zu hohem Masse weggelassen werden konnten, und zwar wurde die Verstärkung für die $\alpha$-Wellen nicht so stark herabgesetzt.

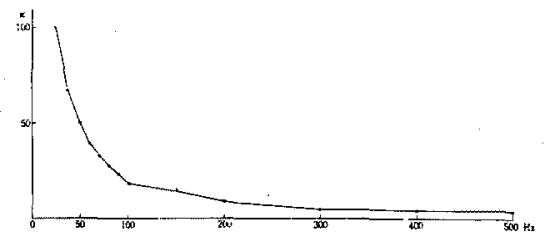

Abb. 2. Verstärkung-Frequenz-Kennlinie des Verstärkers bei der Einsehaltung der Kondensatoren ( $c$ and $c^{\prime}$ ).

Ordinate: Relative Spaunungsverstärkung. Abszisse: Frequenzzahl.

\section{Oszillograph.}

Ich brauchte den Schleifenoszillographen des elektromagnetischen Typus von Y.E.W., Japan, mit Betrachtungs- und Registrierungsvorrichtungen. Die Oszillographschleife hat eine Empfindlichkeit von $10^{-4} \mathrm{~V} / \mathrm{mm}$, eine Eigenschwingung von $1000 \mathrm{~Hz}$. und einen Widerstand von $20 \mathrm{hm}$. Bei diesem Verstärker- Oszillographensystem mit 2 Kondensatoreneinschaltungen ( $\mathrm{C}$ und $\mathrm{C}^{\prime}$ ) und fast 90\% des maximalen Regulierwiderstandes (R) rief eine rechtwinklige elektrische Spannung von $20 \mu \mathrm{V}$ einen Ausschlag von etwa $6 \mathrm{~cm}$ auf dem photographischen Papier hervor.

\section{Reizlicht.}

Die als Lichtreiz angewandte weisse Lichtfläche war kreisrund und $10 \mathrm{~cm}$ im Durchmesser (Gesichtwinkel etwa 0,1 Radian). Die Leuchtdichte der Lichtfläche wurde in zweierlei Weise nämlich durch 
den Wechsel der Lichtquelle, d.h. der elektrischen Lampe und durch die Veränderung der Blendenweite abgestuft.

Beim Versuche mit den helladaptierenden Augen kamen 7 verschiedene Mazda Lampen von 200, 100, 60, 40, 13, 5, und 0,15 Watt100 Volt als Lichtquelle in Anwendung, dagegen beim Versuch mit den vollkommen dunkeladaptierenden Augen reichte nur eine einzige Lampe von 100 Volt- 100 Watt aus, denn die Leuchtdichte der Lichtfläche konnte durch die Blenden abgestuft werden. Mittels dieser Blenden kann man die Leuchtdichte der Lichtfläche sehr umfangreich, nämlich $1-10^{5}$ verändern. Dieser Belichtungsapparat wurde mittels des Luxmeter von Matzda (Sperrschichtphotoelemente) genau geeicht. Als elektrische Quelle zu den Lampen diente ein 100 VoltAkkumulator.

Die Reizdauer wurde in der Weise registriert, dass beim Stromschluss das Signal erschien und beim Stromöffnen verschwand. Es muss hier beachtet werden, dass das Erscheinen des Signals, streng genommen, nicht den realen Anfang des Reizlichtes, sondern den des elektrischen Stromes im Lampenkreis anzeigt. Denn die Lichtstärke der Lampe erreicht sein Maximum niemals sofort beim Stromschluss, sondern kann dazu eine so lange Zeit, wie 0,3 Sek. bei 200 WattLampe, brauchen. Ich habe die Anstiegsform der Lichtstärke einzelner Lampen beim Stromschluss durch das Cs-Photozellen-Gleichstromverstärker-Oszillographensystem untersucht. Das Resultat ist beispielsweise in Abb. 3 dargestellt. Die Geschwindigkeit der Lichtstärkezunahme ist je nach dem Watt der Lampe verschieden, aber die Form derselben stimmt bei allen Lampen im grossen und ganzen überein und lässt sich mit einer exponentiellen Formel ausdrücken, das allererste Stadium ausgenommen. Daher habe ich nur die Anstiegszeit bis zu 90\% der maximalen Lichtstärke in Tab. 3 angegeben.

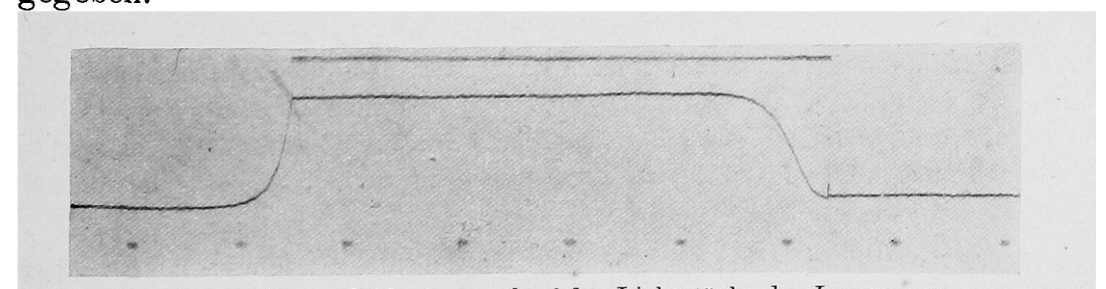

Abb. 3. An- und Abstiegsverlauf der Lichtstärke der Lampe von 100 V-100 W. Die ausgezogene Linie zeigt die Einschaltungsdauer des elektrischen Stromes. Von rechts nach links zu lesen. Zeit in $1 / 5$ Sek. 


\section{E. Registrierung der Reaktionszeit.}

Die Reaktionszeit (R.Z.) wurde nebeneinander mit den $\alpha$-Wellen auf dem photographischen Papier registriert, indem ein elektrischer Berührungsschlüssel von der Versuchsperson (V.P.) durch die Beugebewegung des rechten Zeigefingers geöffnet, sobald sie das Licht empfand. Dieser Schlüssel wurde durch den aus Ebonite und Holz bestehenden Stiel von etwa $1 \mathrm{~m}$ Länge von der V.P. elektrisch isoliert, konnte aber von der V.P. mit nur geringem. Widerstand geöffnet werden.

\section{F. Versuchsperson und Versuchsanordnung.}

Durch diesen ganzen Versuch hindurch hatte Prof. K. Motokawa, Direktor in unserem Institut, ein gesunder Mann von 38 Jahren, die Güte als V.P. zu dienen.

Die V.P. lag in der bequemsten Seitenlage auf einem von der Erde gut isolierten Bette, und wandte möglichst richtig der Lichtfläche ihr Gesicht mit bequem geöffneten Augen zu. Aber kein Fixierpunkt der Gesichtslinie wurde zur Verwendung gebracht, weil er auf das Auftreten der u.Wellen der V.P. hemmend wirkt. Beim Versuch sah die V.P. in körperlicher und geistiger Ruhe binokular die Lichtfläche, die etwa $1 \mathrm{~m}$ entfernt von den Augen auf gleichem Niveau lag. Die Versuche wurden in einen vollkommen dunklen und auch möglichst schallfrei gemachten Zimmer ausgeführt.

Das Reizlicht dauerte gewöhnlich ungefähr 1 Sek., aber die Dauer wurde unter Umständen etwas verlängert, was zur Registrierung der besonders bei langen Reaktionszeiten wie bei sehr schwacher Lichtstärke unbedingt notwendig war. Auf jeden Fall wurde die V.P. dem Lichtreiz wenigstens bis zur Vollendung der Reaktionsbewegung ausgesetzt.

Nachdem der bestimmte Adaptationszustand der Augen erreicht worden war, machte der Experimentator der V.P. mit einem Schallsignal den Versuchsbeginn bekannt, so dass die letztere sich dazu bereit machen konnte. Der Lichtreiz wurde erst dann der V.P. gegeben, wenn es innerhalb 3 Minuten nach dem Anfangsschallsignal an der Betrachtungsvorrichtung des Oszillographen konstatiert war, dass die $\alpha$-Wellen voll entwickelt waren. Da die $u$-Wellen sich spontan verkleinern können, und der Einfluss des bei dieser reduzierten Phase gegebenen Reizes auf die $\alpha$-Wellen schwer erkennbar ist, ist das oben erwähnte Verfahren auch zur Sparsamkeit des photographisehen Papiers notwendig. Ausserdem hat dies Verfahren noch den Vorteil, dass das Intervall zwisehen dem Schallsignal und der Reizgebung beim einzelnen Versuch unregelmässig wird, was zur Vermeidung von etwaiger Antizipation bei der Reaktionszeitmessung dienen kann.

Die Reaktionszeit ist im allgemeinen vielfach von der Aufmerksamkeit der V.P. ahhängig. In diesen Versuchen musste die V.P. sich im psychisch unbefangenen Zustand befinden, um $\alpha$-Wellen voll entwickelt zu erhalten. Daher kann die Reaktionszeit in diesem Zustand etwas länger alsüblich ausfallen. 


\section{Versuchsergibnisse.}

A. Allgemeines.

Ich möchte hier die allgemeine Inspektion über die E.E.G. vorausschicken. Abb. 4 A zeigt das E.E.G. ohne motorische Reaktion.

A.

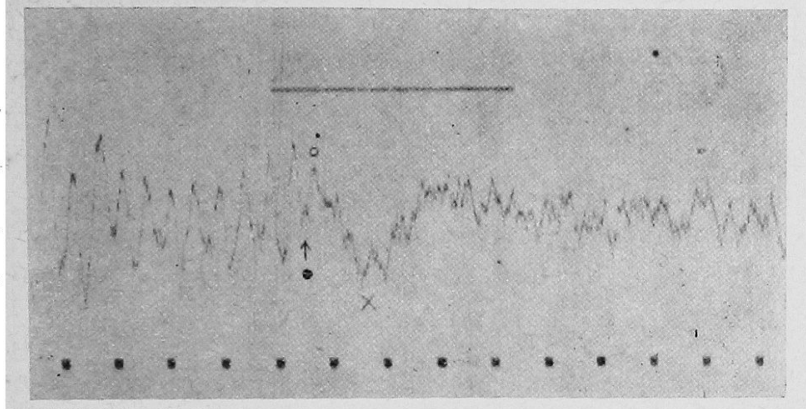

B.

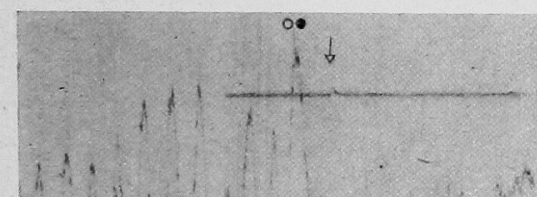

c.

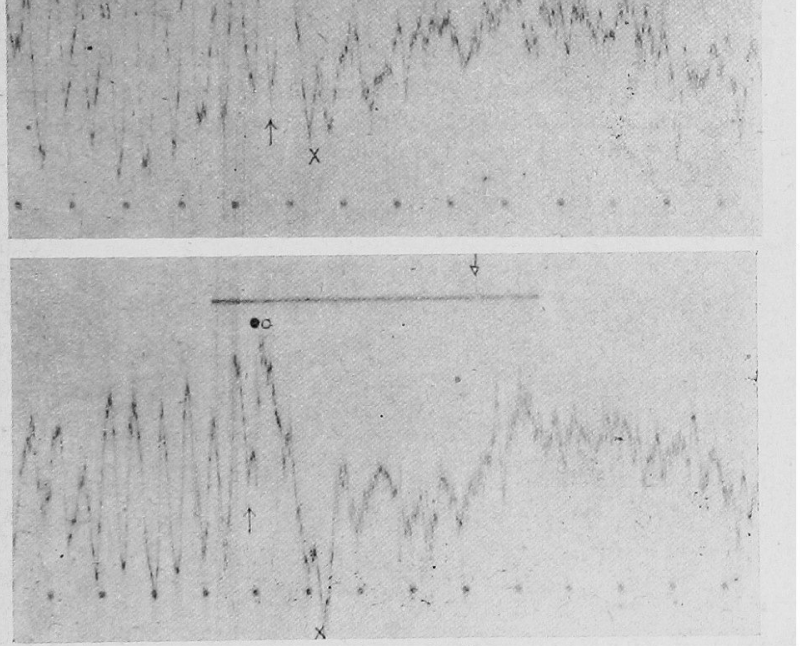

Abb. 4. Elektrenkephalogramme.

A: Bei der Belichtung der Augen allein ohne Reaktionsbewegung,

B : Bei der einfachen Reaktion.

C : Bei der komplizierten Reaktion.

Die ausgezogene Linie zeigt die Belichtungsdauer.

Die Zacke auf der Linie zeigt das Moment der motorischen Reaktion.

Von links nach rechts zu lesen. Zeit in $1 / 5$ Sek.

$$
\underset{\nabla}{\checkmark} \text { : R.Z., } \uparrow \text { : E.L., O: U.L., O: N.G.Z, } \times \text { : P.G.Z. }
$$


Wie man daran bemerken wird, werden die voll entwickelten $\alpha-W e l l e n$, deren Schwingungszahi zwischen $9,5-11,5 \mathrm{~Hz}$. beträgt, in der bestimmten Zeit (von etwa 1,5 Wellenlänge der $\alpha$-Wellen) nach dem Lichtreiz beträchtlich verkleinert, um dann zu verschwinden. Fast gleichzeitig kommen hochfrequente Wellen mit relativ grösseren Amplituden als vorher sowie auch die langsamen grossen Ausschläge der Nullinie zum Vor'schein. Man kann auch in Abb. 5 das gleiche Verhältnis sehen, wobei die auf jede 3,5 Sekunden wiederholt gegebenen Lichtreize hin auftretenden Spannungsschwankungen gezeigt sind. In diesem E.E.G. soll man ferner beachten, dass in ungefähr 0,5 Sek. nach jedem Lichtreize die nach unten gerichteten grossen Ausschläge immer auftreten ( $x$-Zeichen). Diese Ausschläge seien hier als „,Oneffekt" genannt.

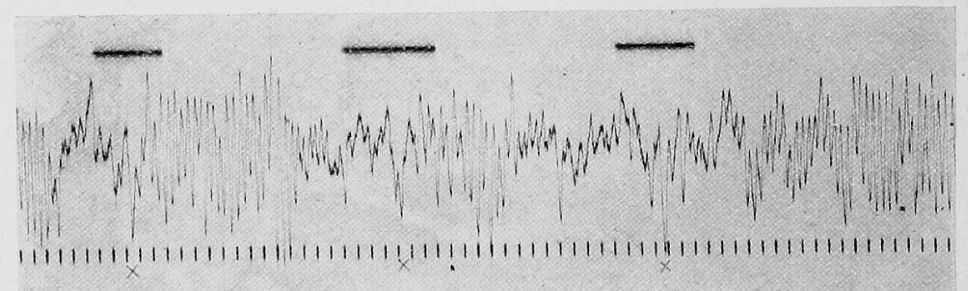

Abb. 5. E.E.G. bei wiederholter Belichtung der Augen mit der ausgedehnten Lichtfläche, welche der Gesichtlinie senkrecht von den Augen.etwa $20 \mathrm{~cm}$ entfernt steht und die Leuchtdichte von $4,5 \times 10^{-5}$ Millilambert hat. Von rechts nach links zu lesen. Zeit in $1 / 5$ Sek. $\times$ : Elektropositiver Gipfel des Oneffektes.

Ich bezeichnete die allerkürzeste Latenz als ,, Einwirkungslatenz" (E.L.), worunter man das Intervall zwischen dem Reizmornent und dem bemerkbaren Beginn irgendeiner Abweichung vom normalen Zustand der Wellen verstehen möge. Aber da es in der Praxis fast unmöglich ist, innerhalb der halben Wellenlänge der $\alpha$-Wellen den Beginn der Abweichung richtig zu bestimmen, so wählte ich den letzten intakten Gipfel der $\alpha$-Wellen als Merkınal (In Abb. 4 mit $\uparrow$ Zeichen dargestellt).

Als Abweichungen können folgende drei Veränderungen angeführt werden, nämlich :

1. der grosse Ausschlag der Nullinie, d.h. das Auftreten des Oneffektes,

2. plötzliche Veränderung der Wellenlänge,

3. Verkleinerung der Amplitude od. Verschwinden der $\alpha$ - 
Wellen.

Unter diesen drei Veränderungen kommt die erste fast immer am frühesten zustande. Daher ist die E.L. im grossen und ganzen als die Latenzzeit für den Oneffekt aufzufassen.

Jasper) bezeichnete das Zeitintervall zwischen dem Reizbeginn und dem letzten bemerkbaren Gipfel der $\alpha$-Wellen als , blocking time." Ich bezeichnete dagegen dasselbe Zeitintervall als „Unterdrückungslatenz" (U.L.), um die letztere von der E.L. zu unterscheiden. Wie schon Jasper ${ }^{6)}$ und Cruikshank ${ }^{2)}$ darauf hinwiesen, war die Bestimmung der U.L. der $\alpha$-Wellen sehr schwierig, denn sie wird durch das Vorhandensein des Oneffektes erschwert.

Auch die Bestimmung der E.L. ist nicht ganz frei von Schwierigkeiten; die $\alpha$-Wellen verändern ihre Amplitude spontan von Zeit zu Zeit und auch die Nullinie kann aus unbekannten Ursachen etwas schwanken. Unter Umständen gingen die $\alpha$-Wellen so allmählich verloren, dass die Bestimmung dieser Latenzzeiten kaum möglich schien, und bei solchen Fällen musste ich auf die Messung verzichten.

Es muss hierbei über den Oneffekt etwas bemerkt werden, der im allgemeinen als diphasische Ausschläge auftritt. Der Vorschlag, welcher in Abb. 4, 6 und 8 nach oben gerichtet und mit o-Zeichen angezeigt ist, entspricht der elektronegativen Veränderung am Hinterhaupt gegen die Stirn, und der nach unten gerichtete mit $\times$ gezeichnete Ausschlag der elektropositiven Veränderung (siehe Abb. 4, 5,6 und 8.).

Der erstere tritt meistens vor der $\alpha$-Wellen-Unterdrückung, also auf die $\alpha$-Schwankung überlagert auf. Der zweite Ausschlag, meistens hier viel deutlicher als der Vorschlag, erreicht sein Maximum in der Regel nach der Unterdrückung der $\alpha$-Wellen. Hier definierte ich die Intervalle zwischen dem Reizbeginn und den beiden Gipfeln als die "negative Gipfelzeit" (N.G.Z.) und die "positive Gipfelzeit" (P.G.Z.) resp. Wenn der erste Gipfel auffallend auftritt, sind die E.L. und die N.G.Z. natürlich leicht festzusetzen und der so bestimmte Wert der E.L. oder der N.G.Z. ist mit ,implicit time " Bartle y s $^{1\rangle}$ vergleichbar.

Die Bestimmung der N.G.Z. und der P.G.Z. war viel leichter als die der E.L. und der U.L., weil die Schwankungen des Oneffektes sehr auffallend waren. Sie sind natürlich durch den Lichtreiz, aber nicht durch die Reaktionsbewegung bedingt, was daraus ohne weiteres zu entnehmen ist, dass diese Schwankungen ohne Reaktionsbewegung des Fingers auftreten. (Vergleiche Abb. $4 \mathrm{~A}$ od. 5 mit Abb. 4B, 4C). 


\section{B. Vergleich der einfachen Reaktionszeit mit der komplizierten Reaktionszeit.}

Hier muss man unter der einfachen Reaktionszeit das Zeitintervall zwischen dem Lichtreiz und der Fingerbewegung verstehen, mit welcher die V.P. (Reagent) dem Lichtreize sofort zu antworten hat. Dagegen bedeutet die komplizierte Reaktionszeit das Intervall zwischen dem Lichreize und der Bewegung, mit der die V.P. dann möglichst rasch reagieren soll, sobald sie die Farbe des dargebotenen Lichtes erkennt. Dabei wurden eine von drei Farben, Weiss, Rot und Blau beliebig dargeboten. Also ist diese komplizierte Reaktionszeit eine Art der sog. Erkennungsreaktionszeit.

Die Versuche wurden innerhalb 3 Minuten nach der Verdunkelung des von einem 100 Volt-60 wattigen Lampe beleuchteten Versuchszimmers ausgeführt. In $\mathrm{Abb} .4 \mathrm{~B}$ und $4 \mathrm{C}$ sind die E.E.G. bei den beiderartigen Reaktionen dargestellt. Bei der Übersicht der beiden E.E.G. zeigt sich keine nennenswerte Verschiedenheit trotz der ganz verschiedenen geistigen Arbeit in beiden Fällen. Das lässt sich von den gesamten Versuchen aussagen.

Die Mittelwerte von U.I., N.G.Z., P.G.Z. und R.Z. beider Reaktionen sind in Tabelle 1 gezeigt, woraus hervorgeht, dass die Mittelwerte der U.L., N.G.Z. sowie P.G.Z. bei beiden Reaktionen miteinander ziemlich genau übereinstimmen.

Bei der einfachen Reaktion war der Mittelwert der U.L. 0,20 Sek. und die kürzeste U.L. 0,11 Sek. Bei der komplizierten Reaktion war der Mittelwert der U.L. 0,19 Sek. und die kürzeste U.L. 0,13 Sek. Die grösste Schwankungsbreite der U.L. bei den beiden Reaktionen war zugleich 0,12 Sek., d.h. gleich der Wellenlänge der $\alpha$-Wellen.

$$
\text { Tabelle } 1 .
$$

Vergleich der einfuchen Reaktionszeit mit der komplizierten Reaktionszeit.

Ziffern in Klammern zeigen die Messungszahl.

Leuchtdichte der Lichtfläche: 25 Millilambert

\begin{tabular}{|c|c|c|c|c|c|}
\hline & & $\begin{array}{c}\text { Unter- } \\
\text { drückungs- } \\
\text { latenz (U.L.) } \\
\text { in Sek. }\end{array}$ & $\begin{array}{c}\text { Negative } \\
\text { Gipfelzeit } \\
\text { (N.G.Z.) } \\
\text { in Sek. } \\
\end{array}$ & $\begin{array}{c}\text { Positive } \\
\text { Gipfelzeit } \\
\text { (P.G.Z.) } \\
\text { in Sek. }\end{array}$ & $\begin{array}{l}\text { Reaktions } \\
\text { zeit } \\
(\text { R.Z.) in Sek. }\end{array}$ \\
\hline \multirow{2}{*}{$\begin{array}{l}\text { Finfache } \\
\text { Reaktion }\end{array}$} & \multirow{2}{*}{$\begin{array}{l}\text { Mittelwerte } \\
\text { Sehwankungs- } \\
\text { breite }\end{array}$} & $0,20(12)$ & $0,22(25)$ & $0,38(25)$ & $0,39(20)$ \\
\hline & & $0,11-0,23$ & $0,18-0,27$ & $0,33-0,42$ & $0,33-0,44$ \\
\hline \multirow{2}{*}{$\begin{array}{l}\text { Komplizierte } \\
\text { Reaktion }\end{array}$} & \multirow{2}{*}{$\begin{array}{l}\text { Mitterwerte } \\
\text { Schwankungs } \\
\text { breite }\end{array}$} & $0,19(10)$ & $0,20(10)$ & $0,36(10)$ & $0,88(\mathrm{I} 0)$ \\
\hline & & $0,13-0,25$ & $0,17-0,22$ & $0,32-0,40$ & $0,71-1,09$ \\
\hline
\end{tabular}


Die E.L. war um 0,04 Sek. im Durchschnitt, d.h. um ungefähr die Halbwellenlänge der $\alpha$-Wellen kürzer als die U.L. in beiden Reaktionen. Bei der einfachen Reaktion betrug der Mittelwert der N.G.Z. 0,22 Sek. und derselbe der P.G.Z. 0,38 Sek., während die entsprechenden Werte bei der komplizierten Reaktion resp. 0,20 Sek. und 0,36 Sek. betrugen.

Die.Differenzen der Mittelwerte von E.L., U.L., N.G.Z. und P.G.Z. zwischen beiden Reaktionen sind klein resp.; genauer betrachtet, sind sie bei der einfachen Reaktion ein wenig kürzer als bei der komplizierten. Aber in Betracht des ziemlich grossen Versuchsfehlers dürfte man diesem geringen Unterschied keine grosse Bedeutung zuschreiben.

Ausserdem sind die elektrischen Bilder bei beiden Reaktion kaum zu unterscheiden. Aus diesen Tatsachen dürfte man schliessen, dass der Farbenunterscheidungs- und Lichtempfindungsvorgang im Gehirn auf das E.E.G., wenigstens seinen Anfangsteil, d.h. bis zur Reaktionszeit fast gleiche Wirkung haben. Die einfache Reaktionszeit ist 0,39 Sek. in Mittelwert, die komplizierte dagegen 0,88 Sek. und die Differenz der beiden also 0,49 Sek., welche der Farbenunterscheidungsvorgang des Hirnes in Anspruch nahm.

\section{Beim Helladaptationszustand.}

Es wurden 7 Stufen von der Leuchtdichte der Lichtfïche d.h. 218, $90,25,10,3.5$ und 0,26 Millilambert (M.L.B.) gewählt, indem als Lichtquelle die elektrischen Lampen von $200,100,60,40,13,5$ und 0,15 Watt zur Anwendung kamen. Wie eingangs erwähnt, steigt die Lichtstärke bei Einschaltung des elektrischen Stromes je nach der Lampe mit verschiedener Geschwindigkeit an, die mit $90 \%$-Zeit in Tabelle 3 ausgedrückt ist.

Die Versuche wurden innerhalb 3 Minuten nach dem Dunkeleintritt aus dem bestimmten Helladaptationszustand durchgefïhrt, wie er sich in einem von 100 Volt- 60 wattigen M a z da- Lampe beleuchteten Versuchszimmer einstellte. Die erhaltenen Werte der E.L., U.L., N.G.Z., P.G.Z., und R.Z. sind in Tabelle 2 als Beispiel dargestellt. Tabelle 3 stellt die mittleren Werte aus einer Reihe der Versuche zusammen, welche mit der oben erwähnten 7 artigen Leuchtdichte angestellt wurden. Die Abb. 6 zeigt 3 E.E.G. bei 218,18 und 0,26 M.L.B.

Das Resultat der Versuche ist in Abb. 7 graphisch dargestellt. Auf der logarithmisch eingeteilten Abszisse ist die Leuchtdichte 


\section{Tabelle 2.}

Versuche beim Helladaptationszustand.

Leuchtdichte der Lichtfläche: 90 M.L.B. (Lichtquelle: $100 \mathrm{~V}-100 \mathrm{~W}$-Lamp).

\begin{tabular}{|c|c|c|c|c|c|}
\hline Datum. Versuch & $\begin{array}{c}\text { Ein- } \\
\text { wirkungs- } \\
\text { latenz (E.L.) } \\
\text { in Sek. }\end{array}$ & $\begin{array}{c}\text { Unter- } \\
\text { drückungs- } \\
\text { latenz (U.L.) } \\
\text { in Sek. }\end{array}$ & $\begin{array}{c}\text { Negative } \\
\text { Gipfelzeit } \\
\text { (N.G.Z.) } \\
\text { in Sek. }\end{array}$ & $\begin{array}{l}\text { Positive } \\
\text { Gipfelzeit } \\
\text { (P.G.Z.) } \\
\text { in Sek. }\end{array}$ & $\begin{array}{l}\text { Reaktions- } \\
\text { zeit (R.Z.) } \\
\text { in Sek. }\end{array}$ \\
\hline 27. III. 1940. 2 & 0,16 & 0,22 & 0,22 & 0,35 & $-0,31$ \\
\hline 28. III. $1940.17 \mathrm{a}$ & 0,21 & 0,34 & 0,29 & 0,35 & 0,38 \\
\hline do. & ? & ? & 0,25 & 0,35 & 0,39 \\
\hline do. & ? & $?$ & 0,18 & 0,38 & 0,38 \\
\hline 30. III. $1940.36 \mathrm{a}$ & 0,18 & 0,25 & 0,20 & 0,35 & 0,33 \\
\hline do. & 0,14 & ? & & 0,35 & 0,39 \\
\hline do. & $?$ & 0,34 & 0,18 & 0,33 & 0,38 \\
\hline Mittelwert & 0,17 & 0,26 & 0,22 & 0,35 & 0,38 \\
\hline
\end{tabular}

Tabelle 3.

Versuche beim Helladaptationszustand.

Ziffern in Klammern zeigen die Messungszahl.

\begin{tabular}{|c|c|c|c|c|c|c|c|}
\hline $\begin{array}{l}\text { Lampe } \\
\text { in } \\
\text { Watt }\end{array}$ & $\begin{array}{l}\text { Leucht: } \\
\text { dichte } \\
\text { in } \\
\text { M.L.B. }\end{array}$ & $\begin{array}{c}90 \%- \\
\text { Zeit in } \\
\text { Millisek. }\end{array}$ & $\begin{array}{c}\text { Ein- } \\
\text { wirkangs- } \\
\text { latenz } \\
\text { (E.L.) } \\
\text { in Sek. }\end{array}$ & \begin{tabular}{|} 
Unter- \\
drüekungs- \\
latenz \\
(U.L.) \\
in Sek.
\end{tabular} & $\begin{array}{c}\text { Negative } \\
\text { Gipfelzeit } \\
\text { (N.G.Z.) } \\
\text { in Sek. }\end{array}$ & $\begin{array}{c}\text { Positive } \\
\text { Gipfelzeit } \\
\text { (P.G.Z.) } \\
\text { in Sek. }\end{array}$ & $\begin{array}{c}\text { Reaktions- } \\
\text { zeit } \\
\text { (R.Z.) } \\
\text { in Sek. }\end{array}$ \\
\hline 200 & 218 & 207 & $0,12(11)$ & $0,24(7)$ & $0,21(14)$ & $0,37(15)$ & $0,37(15)$ \\
\hline 100 & 90,0 & .127 & $0,17(4)$ & $0,26(4)$ & $0,22(6)$ & $0,35(7)$ & $0,37(7)$ \\
\hline 60 & 24,8 & 96,9 & $0,19(4)$ & $0,23(4)$ & $0,22(4)$ & $0,37(6)$ & $0,38(6)$ \\
\hline 40 & 18,0 & 68,2 & $0,15(4)$ & $0,20(5)$ & $0,19(9)$ & $0,35(9)$ & $0,35(9)$ \\
\hline 13 & 10,0 & 63,1 & $0,15(6)$ & $0,21(3)$ & $0,20(7)$ & $0,36(8)$ & 0,34 (8) \\
\hline 5 & 3,5 & 49,6 & $0,13(6)$ & $0,22(5)$ & $0,21(7)$ & $0,38(7)$ & $0,38(7)$ \\
\hline 0,15 & 0,26 & 55,1 & $0,22(5)$ & $0,30(5)$ & $0,27(4)$ & $0,41(6)$ & $0,42(6)$ \\
\hline
\end{tabular}

(M.L.B.) und auf der Ordinate die Zeit in Millisek. aufgetragen. Wie in Tab. 3 und Abb. 7 ersichtlich ist, bewegen sich die mittleren Werte der R.Z. unter den geschilderten Versuchsbedingungen zwischen 0,34 Sek. bei 10 M.L.B. (13 Watt- Lampe) und 0,42 Sek. bei 0,26 M.L.B. $(0,15$ W-L.). Es erscheint, als ob die R.Z. zu der Lichtstärke in nicht so einfạcher Beziehung stehe, aber es ist auch möglich, dass eine andere Ursache als Lichtstärke dieser Unregelmässigkeit zugrundeliegt, worauf ich unten wieder zurückkommen werde.

Wie aus der Abb. 7 ersichtlich ist, verlaufen alle Kurven (E.L.-, U.L.-, N.G.Z.-, P.G.Z.-, und R.Z.- Kurven) im grossen und granzen miteinander parallel, vor allem fäjlt die R.Z.- Kurve mit der P.G.Z.Kurve, fast unabhängig von der Reizstärke, also auch von der Art der Lampe, zusammen.

Wenn die beiden Gipfel des Oneffektes deutlich in Erscheinung 


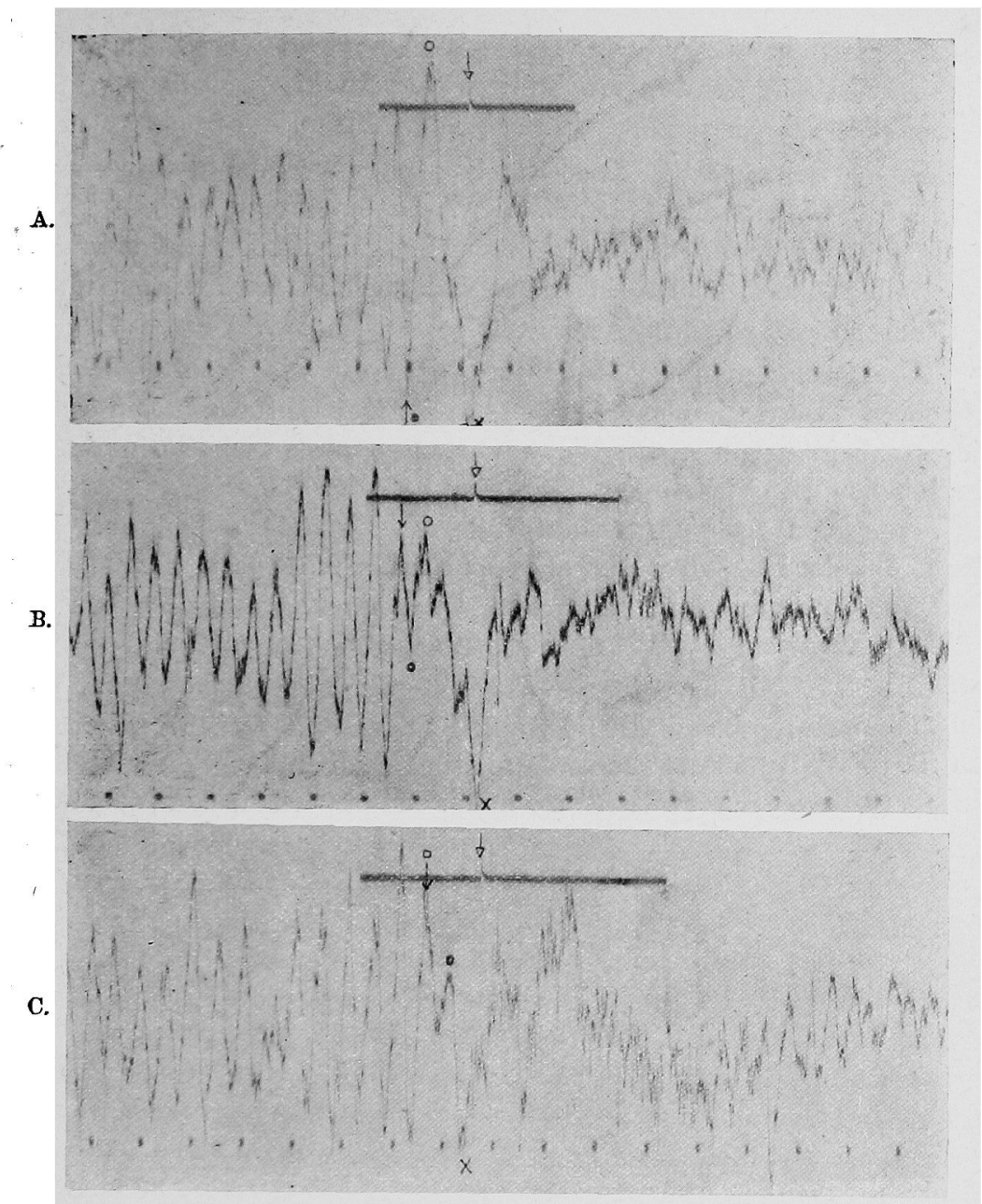

Abb. 6. E.E.G. bei Belichtung der helladaptierten Augen mit den Lichtflächen von A : 218 M.L.B., B: 18 M.L.B., C: 0,26 M.L.B., Von links nach rechts zu lesen. Zeit in $1 / 5$ Sek. $\frac{1}{\nabla}$ : R.Z., $\uparrow:$ E.L., ๑ : U.L., O: N.G.Z., $\times:$ P.G.Z.

treten, bleiben dieZeitintervalle $\mathrm{zwischen}$ den beiden, unabhängig von der Lịchtstärke, fast konstant (0,16 Sek.). Wenn einer von den beiden Gipfeln, insbesondere der elektronegative, auch bei einem schwachen Reize oft vermisst wurde, kam das gleichzeitige Fehlen der beiden doch niemals bei diesen Versuchen vor. Die $\alpha$-Wellen-Unterdrükkung trat meistens an dem elektronegativen Gipfel des Oneffektes od. 
noch ein wenig später auf. Die $\alpha$-Wellen verkleinerten sich. bald plötzlich, bald allmählich, um dann zu verschwinden. Die $\alpha$-Wellen können die R.Z. überdauern, wenn sie auch stark unterdrückt sind.

Es ist merkwürdig, dass alle Kurven, der Leuchtdichte von 24 M.L.B. (60 W.-L.) und 18 M.L.B. (40 W.-L.) entsprechend, gemeinsam nebeneinander stark knicken. Dieser Knick der R.Z.- Kurven wider-' spricht der bekannten Tatsache, dass sich die R.Z. mit der abnehmenden Lichtstärke monoton verlängert.

Aber wenn man berïcksichtigt, dass die als Lichtquelle verwendeten Lampen je verschiedene Anstiegszeitfaktoren haben, so ist dieses scheinbar unverständliche Verhalten ohne Schwierigkeit zu verstehen: Die R.Z. muss nicht nur von der Reizstärke, sondern auch von dem Anstiegszeitfaktor der Lichtstärke abhängen. Die Beziehung der R.Z.- sowie der anderen Kurven zum Anstiegszeitfaktor der Lichtstärke ist ohne weiteres zu entnehmen, wenn die oben erwähnten Kurven mit der 90\%-Zeitkurve verglichen werden.

Die 90\% Zeitkurve der Leuchtdichte der Lichtfläche nimmt mit der abnehmenden Leuchtdichte von 218 M.L.B. (200 W.-L.) bis zu 3,5 M.L.B. ( 5 W.-L.) zuerst rasch, dann allmählich ab, um weiter bei 0,26 M.L.B. (0,15 W.-L.) etwas zuzunehmen. Man wird ersehen, dass auch

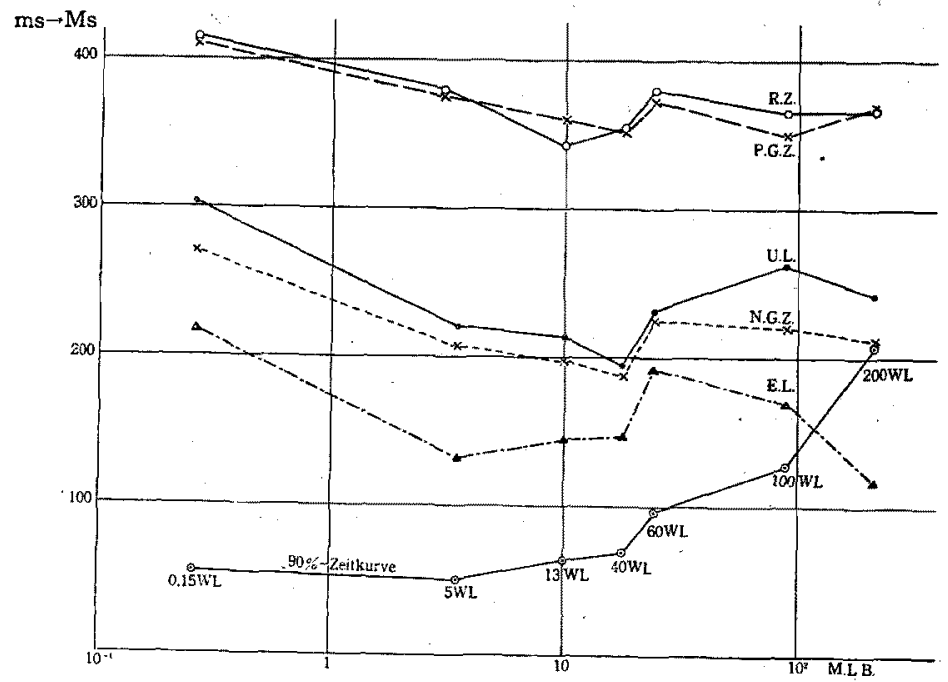

Abb. 7. Die Abhängigkeit der Reaktionszeit und der Latenzzeiten von der Leuchtdichte beim Helladaptationszustand.

Ordinate: Zeit in Millisek.

Abszisse: Leuchtdichte der Lichtfläche in M.L.B. 
diese Kurve beträchtliche Knickungen bei 24,8 M.L.B. ( 60 W.-L.) und 1,8 M.L.B. ( 40 W.-L.) hat. Diese Knicke entsprechen gerade denjenigen der R.Z.-Kurve und der anderen, was darauf hinweist, dass die erwähnte Unregelmässigkeit der R.Z.- Kurve und der anderen durch den Anstiegszeitfaktor bedingt ist.

Definiert man nun die mittlere Zunahmegeschwindigkeit der Lichtstärke folgendermassen: $\log \left(\mathrm{I} / \mathrm{I}_{0}\right) / 90 \%$-Zeit, wo $\mathrm{I}$ und $\mathrm{I}_{\mathrm{o}}$ $(=0,001$ M.L.B. $)$ die maximale und die schwellige Leuchtdichte resp. sind, so ergeben sich folgende Werte für einzelne Lampen als ihre relativen Anstiegszeitfaktoren: 26:39:41:63:64:72:44 für 200, $100,60,40,13,5$ und 0,15 Watt- Lampe resp.

Im allgemeinen werden die R.Z. und die anderen Latenzzeiten mit der Zunahme der Lichtstärke und des Anstiegszeitfaktors verkürzt. Die Tatsache, dass die R.Z. und die anderen bei 60 Watt-Lampe trotz der grösseren Lichtstärke länger als bei 40 Watt-Lampe sind, beruht auf dem Umstand, dass die Lichtstärke bei jenen von der bei diesen nicht so verschieden ist, aber dass der Anstiegszeitfaktor stark verschieden ist ( 60 W.-L. : 40 W.-L. $=41: 63$ ). Die Differenz zwischen der R.Z. sowie den anderen bei 60 W.-L. und den bei 100 W.-L. od. 0,15

- W.-L. muss dagegen hauptsächlich auf dem Intensitätsunterschied der Lampen beruhen, weil diese Lampen einander ganz nahe Anstiegszeitfaktoren haben (100 W.-L. : 60 W.-L. : 0,15 W.-L. $=39: 41: 44$ ).

Es ergibt sich also, dass die bioelektrischen Erscheinungen und die Reaktionszeit des Gesichtsinns in gleicher Weise von der Lichtstärke und dem Anstiegszeitfaktor des Lichtreizes abhängen.

Diese Tatsache weist ferner darauf hin, dass die bioelektrischen und die motorischen Reaktionen auf Lichtreize in zeitlich bestimmter Beziehung stehen, und auch dass schon das Eintreten der afferenten Impulse in die Grosshirnrinde sowohl von der Reizstärke als auch von der Steilheit des Stärkeanstieges abhängt. Dies stimmt mit der am Tiere schon bekannten Tatsache überein. Vor allem muss hier beachtet werden, dass.W ang11) in dieser Hinsicht über den Einfluss des Anstiegszeitfaktores des Lichtreizes auf Elektroretinogramme des. Frosches mitgeteilt hat.

\section{Beim välligen Dunkeladaptationszustand.}

Im Gegensatz zu den Versuchen beim Helladaptationszustand wurde die einzige elektrische Lampe von $100 \mathrm{Watt}$ hier als Lichtquelle verwendet, indem die Leuchtdichte der Lichtfläche nur durch 2 Blend- 
en abgestuft wurde. Also blieb die Steilheit des Stärkeanstieges des Lichtreizes, d.h. der Anstiegszeitfaktor hier unverändert. Die Versuche wurden mit Leuchtdichte unter 2 M.L.B. nach Aufenthalt von 1 Stunde im vollkommen verdunkelten Versuchszimmer ausgeführt.

Die schwächste Leuchtdichte $\left(1,3 \cdot 10^{-6}\right.$ M.L.B.) entsprach hier ungefähr der Reizschwelle des Gesichtssinnes in diesem Adaptationszustand.

A.

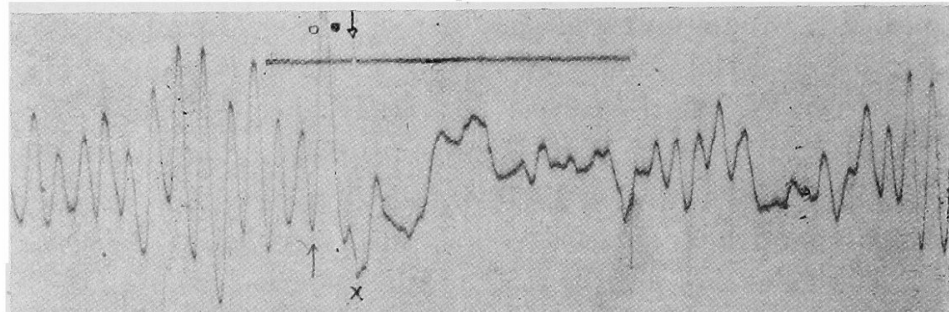

B.
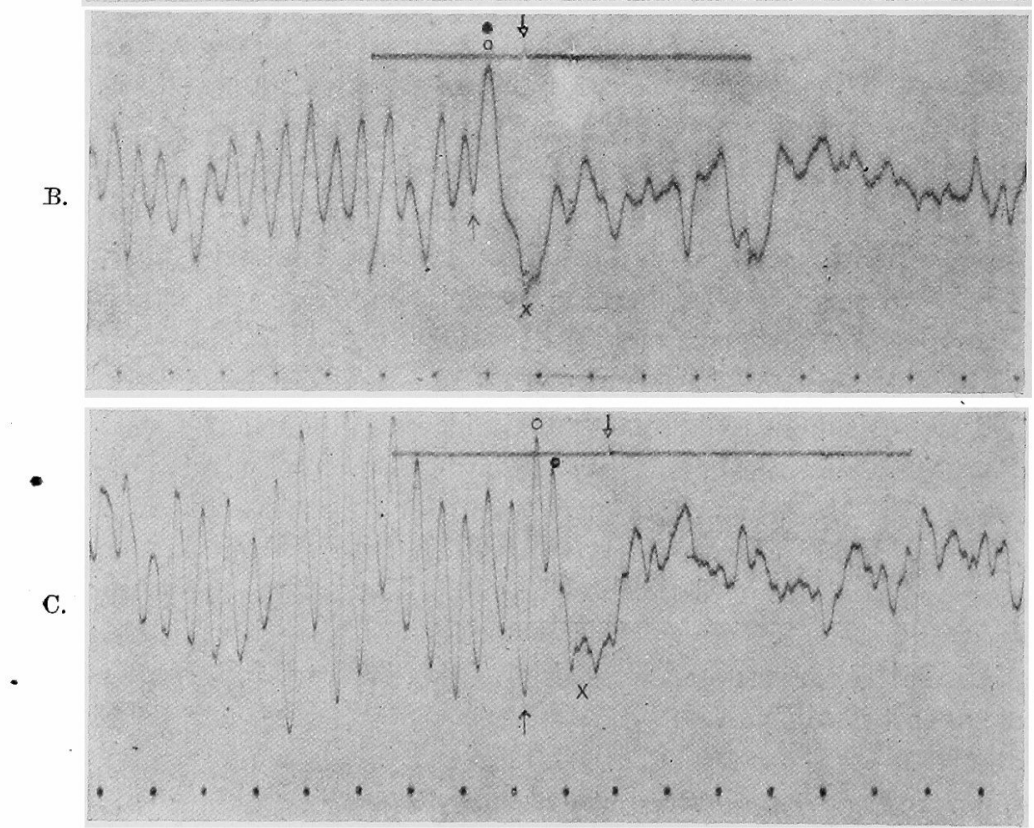

Abb. 8. E.E.G. bei Belichtung der dunkeladaptierenden Augen. Leuchtdichte der Lichtfläche, A: 0,20 M.I.B., B : 1,8 $10^{-4}$ M.L.B., C : 5,2 $10^{-6}$ M.L.B.. Von links nach rechts zu lesen. Zeit in $1 / 5$ Sek. $\frac{1}{\nabla}$ : R.Z., $\uparrow:$ E.L., $\bullet:$ U.L., O: N.G.R., $\times:$ P.G.Z. 
Die Abb. 8 zeigt 3 E.E.G. bei $0,2,1,8 \cdot 10^{-4}$ und $5,2 \cdot 10^{-6}$ M.L.B. Man sieht an dieser Abb., dass wegen der Kondensatoreneinschaltung. des Verstärkers an diesen E.E.G. die hochfrèquenten Wellen ausbleiben, und dass die Unterdrückung der $\alpha$-Wellen sowie der Oneffekt leichter als bisher gelesen werden können. Tab. 4 stellt die mittleren Werte der R.Z., U.L., N.G.Z. und P.G.Z. zusammen und in Abb. 9 ist die Abhängigkeit der R.Z. und der anderen Latenzzeiten von der Leuchtdichte in gleicher Weise wie in Abb. 7 dargestellt.

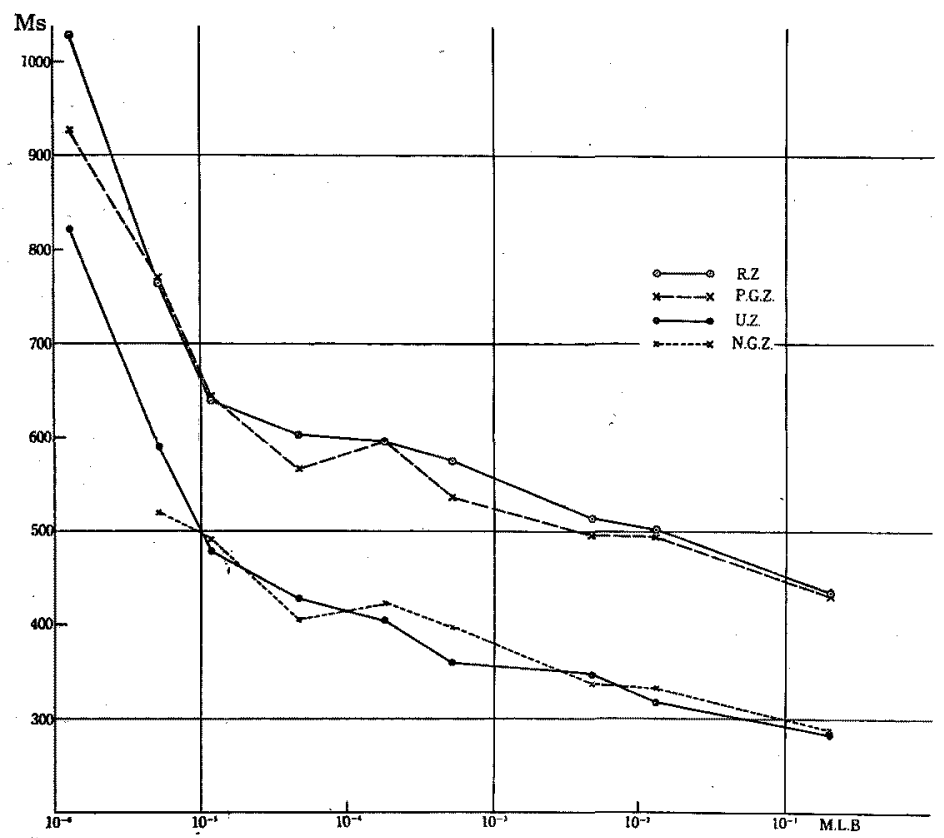

Abb. 9. Die Abhängigkeit der Reaktionszeit und der Latenzzeiten von der Leuchtdichte beim Dunkeladaptationszustand. Ordinate: Zeit in Millisek..

Abszisse: Leuchtdichte der Lichtfläche in M.L.B..

Es zeigt sich, dass die R.Z., U.L., N.G.Z. und P.G.Z. zu der Leuchtdichte in einer einfachen logarithmischen Beziehung stehen, und dass alle Kurven miteinander fast parallel verlaufen. Alle Kurven erscheinen je einen Knick bei 1,17.10 ${ }^{-5}$ M.L.B. zu haben. Bei schwächerer Lichtstärke als diese verlaufen die Kurven viel steiler, mit anderen Worten: Die R.Z. und die anderen Latenzzeiten nehmen mit der abnehmenden Lichtstärke viel rascher zu und erreichen die grössten Werte bei der Schwellenintensität. Aus Tab. 4 ergibt sich, dass die 
Differenzen zwischen der U.L. (od. N.G.Z.) und R.Z. (od. P.G.Z.), unabhängeg von der Leuchtdichte, fast konstant bleiben, dass die R.Z. und die P.G.Z. auch hier, wie beim Helladaptationszustand, zeitlich zusammenfallen, und dass der Anfang der $\alpha$-Wellen-Unterdrückung durchschschnittlich auf gleiche Weise mit dem elektronegativen Gipfel des Oneffektes übereinstimmt. In Abb. 9 verlaufen also die R.Z. und die U.L.-Kurven mit der P.G.Z.-und N.G.Z.-Kurven resp. übereinander.

Tabelle 4.

Versuche beim völligen Dunkeladaptationszustand. Ziffern in Klammern zeigen die Messungszahl.

\begin{tabular}{c|c|c|c|c}
\hline $\begin{array}{c}\text { Leuchtdichte } \\
\text { in M.L.B. }\end{array}$ & $\begin{array}{c}\text { Unterdrückungs } \\
\text { latenz(U.L.) } \\
\text { in Sek. }\end{array}$ & $\begin{array}{c}\text { Negative } \\
\text { Gipfelzeit } \\
\text { (N.G.Z.) in Sek. }\end{array}$ & $\begin{array}{c}\text { Positive } \\
\text { Gipfelzeit } \\
\text { (P.G.Z.) in Sek. }\end{array}$ & $\begin{array}{c}\text { Reaktionszeit } \\
\text { (R.Z.) in Sek. }\end{array}$ \\
\hline $2,0.10-1$ & $0,28(7)$ & $0,29(14)$ & $0,43(14)$ & $0,43(14)$ \\
$1,3 \cdot 10-2$ & $0,32(5)$ & $0,33(6)$ & $0,49(6)$ & $0,50(7)$ \\
$4,7 \cdot 10-3$ & $0,35(6)$ & $0,34(6)$ & $0,50(7)$ & $0,51(8)$ \\
$5,2.10-4$ & $0,36(6)$ & $0,40(7)$ & $0,55(8)$ & $0,57(8)$ \\
$1,8 \cdot 10-4$ & $0,40(8)$ & $0,42(8)$ & $0,60(8)$ & $0,60(8)$ \\
$4,7.10-5$ & $0,43(5)$ & $0,40(6)$ & $0,56(8)$ & $0,60(6)$ \\
$1,17 \cdot 10-5$ & $0,47(4)$ & $0,49(4)$ & $0,64(4)$ & $0,64(6)$ \\
$5,2 \cdot 10-6$ & $0,59(6)$ & $0,52(3)$ & $0,77(6)$ & $0,76(6)$ \\
$1,3 \cdot 10-6$ & $0,89(7)$ & & $0,93(7)$ & $1,04(8)$ \\
& & & &
\end{tabular}

Das Resultat, dass der Anfang der $\alpha$-Wellen-Unterdrückung und auch der erste Gipfel des Oneffektes um 0,18 Sek. im Durchschnitt bei allen Fällen früher als die Fingerreaktion sowie der zweite Gipfel auftreten, ist bedeutungsvoll im Vergleich mit der Tatsache, dass die Differenz zwischen der R.Z. und der Empfindungszeit Fröhlichs"), d.h. der motorische Anteil der R.Z., in die gleiche Grössenordnung fällt.

Bei sehr schwacher Lichtstärke, d.h. in der Nähe der Reizschwelle des Gesichtssinns werden sowohl die $\alpha$-Wellen-Unterdrückung als auch der Oneffekt, insbesondere der elektronegative Gipfel kleiner und undeutlicher als beim stärkeren Reize, und die Schwankung der Werte für die R.Z. ist dementsprechend auffallender.

\section{Diskussion.}

Zwei merkwürdige diphasische Ausschläge (Oneffekt) lassen sich auf Lichtreiz hin ausser den Eigenrhythmus der Grosshirnrinde $(\alpha-$ Wellen) beobachten. Der erste Gipfel entspricht der elektronegativen Veränderung des Hinterhauptes gegen die Stirn, und der zweite der. elektropositiven. Diese Gipfel werden bei dem Kontrollversuche weder durch die Fingerbewegung allein noch durch die Erregung des motorischen Zentrums des Fingers beeinflusst, und treten sowohl bei 
der einfachen Reaktion als auch bei der komplizierten Reaktion mit einer bestimmten Latenzzeit nach dem Lichtreiz auf. Bei meiner Untersuchung scheint dieser Effekt, insbesondere der elektronegative Gipfel in der Nähe des Schwellenreizes unter Umständen zu fehlen. Dies Verhültnis ist derart aufzufassen, dass bei derartigen sehr schwachen Reizen die auftretende Spannung zu schwach ist, um sich mit meiner Untersuchungsmethode nach weisen zu lassen. Es muss noch erwähnt werden, dass die P.G.Z. immer ziemlich genau mit der einfachen Reaktionszeit zusammenfällt. Diese Úbereinstimmung ist sehr bemerkenswért und kann von grosser physiologischer Bedeutung sein, aber der Mechanismus ist vorläufig noch ganz unklar. Denn der Ursprung des Oneffektes ist noch unklar geblieben, obgleich derartige Ströme schon am Tiere beobachtet und auch beim Menschen von Jasper ${ }^{5)}$, Cruikshank ${ }^{2)}$ und anderen konstatiert worden sind. Um das Wesen dieser Ströme beim optischen Reizversuche festzustellen, muss man natürlich ausser dem Grosshirn die Teilnahme anderer Organe, wie z.B. Netzhaut, A ugenmuskeln, optischer Nerven u.s.w. am E.E.G. berücksichtigen, so dass die Aufklärung dieser Ströme künftigen Untersuchungen überlassen werden muss.

Es ist herhorzuheben, dass die Lichtstärke und der Anstiegszeitfaktor die E.L., U.L., N.G.Z., P.G.Z. und R.Z. in gleicher Weise beeinflussen. Mit anderen Worten: Die zeitliche Differenz zwischen der R.Z. und den Latenzzeiten der bioelektrischen Veränderungen des Hirns bleibt, unabhängig von den Reizbedingungen, beinahe konstant. Andererseits haben die körperlichen Reaktionsbewegungen an sich auf die bioelektrischen Latenzzeiten keinen Einfluss, wie oben erwähnt.

Diese Tatsache weist darauf hin, dass die erwähnten bioelektrischen Veränderungen mit der Lichtempfindung in engem Zusammenhang stehen. Vogelsan g ${ }^{10)}$ berichtet, dass mit der Abnahme der Reizstärke die R.Z. zunimmt, und dass bei motorischer Einstellung der V.P. diese Zunahme der R.Z. allein auf der Zunahme der Empfindungszeit beruht, während der motorische Anteil der R.Z. unverändert bleibt. Vergleicht man also mein Resultat mit dem Vogelsangschen, so ergibt sich, dass die Differenz (0,18 Sek bei Dunkeladaptationszustand) zwischen der R.Z. und der U.L. (oder N.G.Z.) und der von Vogelsang gemessene motorische Anteil der R.Z. nicht nur von der Lichtstärke unabhängig sind, sondern auch in die gleiche Grössenordnung fallen. Daher liegt die folgende Annahme nahe, dass der Unterdrückungsbeginn der $\alpha$-Wellen bzw. der elektronegative Gipfel mit der subjektiven Lichtempfindung zeitlich übereinstimmt. 


\section{Zusammenfassung.}

1. Die zeitliche Beziehung der Reaktionszeit zu den bioelektrischen Veränderungen der Grosshirnrinde wurde bei der Hinterhaupt Stirn-Ableitung und mittels des 4-stufigen Verstärkers und des Schleifenoszillographen untersucht.

2. Auf Belichtung des Auges hin tritt eine diphasische Spannungsveränderung ein, die dem bei Tierversuchen bekannten sog. Oneffekt zu entsprechen erscheint.

3. Unterdrückung der $\alpha$-Wellen fängt fast immer mit dem elektronegativen Gipfel des Oneffektes übereinstimmend an.

4. Der zweite Gipfel des Oneffektes stimmt ziemlich gut mit der Reaktionszeit des rechten Zeigefingers überein.

5. Die Reaktionszeit wird mit der Zunahme der Lichtstärke und der Anstiegsgeschwindigkeit derselben kürzer, und dasselbe Verhalten zeigen andere Latenzzeiten der bioelektrischen Erscheinungen.

Die Reaktionszeit-Lichtstärke-Kurve verläuft mit den Latenzzeit-Lichtstärke-Kurven immer parallel, was darauf hinweist, dass die Differenzen zwischen der Reaktionszeit und den Latenzzeiten, unabhängig von der Lichtstärke, konstant bleiben. Vor allem stimmt die Differnz zwischen der Reaktionszeit und der Latenzzeit der $\alpha$-WellenUnterdrückung mit derjenigen zwischen der Reaktionszeit und der Empfindungszeit überein.

6. Beider komplizierten Reaktion ist die Reaktionszeit natürlich länger als bei der einfachen, aber die elektrischen Bilder in beiden Fällen sind kaum zu unterscheiden.

Am Schluss spreche ich Herrn Prof. Dr. K. Matokaw für seine freundliche Leitung und Unterstützung bei dieser Arbeit meinen besten Dank aus.

\section{Literatur.}

(1) Bartley, S.H., Am. J. Physiol., 1934, 108, 397.

(2) Cruikshank, R.M., J. Exp. Psychol, 1937, 21, 625.

(3) Durup, G. et A. Fessard, Ann. Psychol, 1935, 36, 1.

(4) Jasper, H.H., Cold Spring Habor Symp. Quant. Biol., 1936, 4, 320.

(5) Jasper, H.H. and H.L. Andrews, Arch. Neurol. Psychiat., 1936, 39, 96.

(6) J asper, H.H. and R.M. Cruikshan k, J. Gen. Psychol., 1937, 17, 29.

(7) Fröhli ch, Fr. W., Die Empfindungszeit, Jena 1929, S. 144 und 239.

(8) Ito, G. und K. Kaketa, Tohoka J. Exp. Med., 1937, 30, 546.

(9) Loomis, A.L., E.N. Harvey and G. Hobart, J. Exp. Psychol., 1930, $19,249$.

(10) Vogels a ng, K., Pflügers Arch., 1925, 208, 93.

(11) W ang, G.H. and T.W. L u, Chinese J. Physiol., 1937, 11, 471. 